\title{
Electron-Hole Tunneling Revealed by Quantum Oscillations in the Nodal-Line Semimetal HfSiS
}

\author{
M. R. van Delft, ${ }^{1,2}$ S. Pezzini, ${ }^{1,2,}$ T. Khouri, ${ }^{1,2}$ C. S. A. Müller, ${ }^{1,2}$ M. Breitkreiz, ${ }^{3,4}$ L. M. Schoop, ${ }^{5}$ \\ A. Carrington, ${ }^{6}$ N. E. Hussey, ${ }^{1,2}$ and S. Wiedmann ${ }^{1,2, \dagger}$ \\ ${ }^{1}$ High Field Magnet Laboratory (HFML-EMFL), Radboud University, Toernooiveld 7, Nijmegen 6525 ED, Netherlands \\ ${ }^{2}$ Radboud University, Institute for Molecules and Materials, Nijmegen 6525 AJ, Netherlands \\ ${ }^{3}$ Instituut-Lorentz, Universiteit Leiden, P.O. Box 9506, 2300 RA Leiden, Netherlands \\ ${ }^{4}$ Dahlem Center for Complex Quantum Systems and Fachbereich Physik, Freie Universität Berlin, 14195 Berlin, Germany \\ ${ }^{5}$ Department of Chemistry, Princeton University, Princeton, New Jersey 08544, USA \\ ${ }^{6} \mathrm{H}$. H. Wills Physics Laboratory, University of Bristol, Tyndall Avenue, Bristol BS8 1TL, United Kingdom
}

(Received 12 June 2018; published 18 December 2018)

\begin{abstract}
We report a study of quantum oscillations in the high-field magnetoresistance of the nodal-line semimetal HfSiS. In the presence of a magnetic field up to $31 \mathrm{~T}$ parallel to the $c$ axis, we observe quantum oscillations originating both from orbits of individual electron and hole pockets, and from magnetic breakdown between these pockets. In particular, we reveal a breakdown orbit enclosing one electron and one hole pocket in the form of a "figure of eight," which is a manifestation of Klein tunneling in momentum space, although in a regime of partial transmission due to the finite separation between the pockets. The observed very strong dependence of the oscillation amplitude on the field angle and the cyclotron masses of the orbits are in agreement with the theoretical predictions for this novel tunneling phenomenon.
\end{abstract}

DOI: 10.1103/PhysRevLett.121.256602

The advent of topological semimetals (TSMs) created new opportunities to search for relativistic quasiparticles and novel macroscopic quantum phenomena [1,2]. Two examples of TSMs are Dirac semimetals, which possess fourfold degenerate band crossings in momentum space, and Weyl semimetals (WSM), in which the spin degeneracy is lifted. In the latter case, the band crossings are referred to as Weyl points. These points have opposite chirality, leading to various phenomena such as the so-called chiral anomaly in the bulk and Fermi arcs on the surface [1-6]. Within the group of WSMs, there is a class of materials referred to as type-II WSMs with a band structure in which pairs of electron and hole pockets coexist over a range of energy with a topologically protected crossing. For these materials, a new type of quantum oscillation has been theoretically predicted, that results from tunneling between adjacent electron and hole pockets, which has been termed "momentum-space Klein tunneling" [7]. Until now, this particular effect has not been observed either in type-II WSMs or in other classes of TSMs.

In this Letter, we report on quantum oscillations in the nodal-line semimetal (NLSM) HfSiS, demonstrating a "figure of eight" orbit between an adjacent electron pocket $\beta$ and hole pocket $\alpha$, coupled by field-induced tunneling (i.e., magnetic breakdown), with a characteristic frequency $f_{\beta}-f_{\alpha}$. This orbit appears with increasing magnetic field and is only present if the field is almost exactly parallel to the $c$ axis and has a cyclotron mass which is the sum of the individual masses of the two pockets. These results match the expected signatures of the momentum-space Klein tunneling when a finite gap separates the pockets. In the theoretical proposal, Ref. [7], this gap is realized by tuning the Fermi energy $E_{F}$ and/or the tilt angle, while in our case it is due to spin-orbit coupling and is always present. By further increasing the magnetic field, we find highfrequency oscillations that form a complex frequency spectrum and correspond to tunneling across multiple energy gaps, as observed in $\mathrm{ZrSiS}$ [8].

Nodal-line semimetals are Dirac materials in which conduction and valence bands touch each other along a closed trajectory inside the Brillouin zone instead of at discrete nodes in momentum space [9]. Experimentally verified material systems belonging to the class of NLSMs include $\mathrm{PbTaSe}_{2}$ [10], $\mathrm{PtSn}_{4}$ [11] and compounds with a general formula of $X \operatorname{Si} Y(X=\mathrm{Zr}$, Hf and $Y=\mathrm{S}, \mathrm{Se}, \mathrm{Te})$ that are of particular interest, as they reveal a number of distinct features predominantly investigated in the Dirac NLSM ZrSiS [12-19]. Importantly, in the absence of spinorbit coupling (SOC), the only bands that cross $E_{F}$ in $\mathrm{ZrSiS}$ have a Dirac-like dispersion, with the crossing points forming a line node in the Brillouin zone and giving rise to a cage-like Fermi surface [13].

Until now, HfSiS has been much less investigated than other members of the $\mathrm{ZrSiS}$ family [20-22]. As with all the compounds with a general formula of $X \mathrm{Si} Y$, HfSiS has a $\mathrm{PbFCl}$-type structure (tetragonal space group $\mathrm{P} 4 / \mathrm{nmm}$ ) with layers of $\mathrm{Hf}$ and $\mathrm{S}$ sandwiched between $\mathrm{Si}$ square nets extending in the $a-b$ plane. Its electronic band structure, 


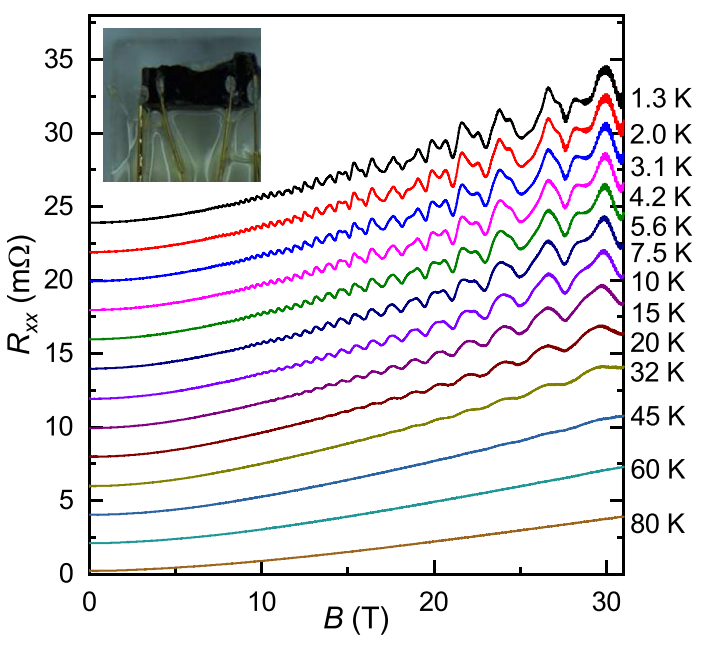

FIG. 1. Longitudinal resistance $R_{x x}$ as a function of the magnetic field for temperatures between 1.3 and $80 \mathrm{~K}$ up to $31 \mathrm{~T}$ with $\mathbf{B} \| c$. Each successive curve is offset by $2 \mathrm{~m} \Omega$ for clarity. The inset shows an image of a typical HfSiS single crystal with attached current and voltage probes.

calculated by density functional theory (DFT), is displayed in Fig. S1 in the Supplemental Material [23] and shows linearly dispersive bands over a large energy range $(\sim 2 \mathrm{eV}$ around $E_{F}$ ). The Fermi surface of HiSiS (presented in a three-dimensional representation in Fig. S2 in the Supplemental Material [23]) contains adjacent electron and hole pockets, realizing an analogue of the Fermi surface of Weyl type-II materials. However, due to spinorbit coupling, a gap separates the pockets in the Dirac spectrum, with a calculated size 2-3 times larger than found in $\mathrm{ZrSiS}$ [28]. Indeed, the size of this gap is such that tunneling between adjacent orbits is not expected up to very high magnetic fields.

Figure 1 shows a series of resistance sweeps as a function of the magnetic field up to $31 \mathrm{~T}$ on a HfSiS single crystal at different temperatures between 1.3 and $80 \mathrm{~K}$. The magnetic field is applied parallel to the $c$ axis. As seen in the figure, HfSiS displays Shubnikov-de Haas $(\mathrm{SdH})$ oscillations with multiple frequencies superimposed on a positive magnetoresistance (MR). Above $15 \mathrm{~T}$, additional high-frequency oscillations are observed, which we shall return to later.

In Fig. 2, we perform a fast Fourier transform (FFT) analysis of the $1.3 \mathrm{~K}$ sweep in Fig. 1. Fig. 2(a) illustrates the FFT amplitude in the low-frequency part of our spectrum that has been generated from data between 5 and $31 \mathrm{~T}$. The observed frequencies, $f$, in the FFT spectra are related to the extremal area $A_{F}$ of the individual pockets via the Onsager relation $f=(\hbar / 2 \pi e) A_{F}$ [29].

In this figure, a number of clearly resolved peaks are visible. The two largest amplitude peaks at 264 and $473 \mathrm{~T}$ we label as $\alpha$ and $\beta$ respectively. Several other peaks can be identified as the second harmonic of $\alpha$ or as the sum and difference of $\alpha$ and $\beta . f_{\alpha}$ was reported previously [22]. The origin of these orbits can be deduced by comparing the
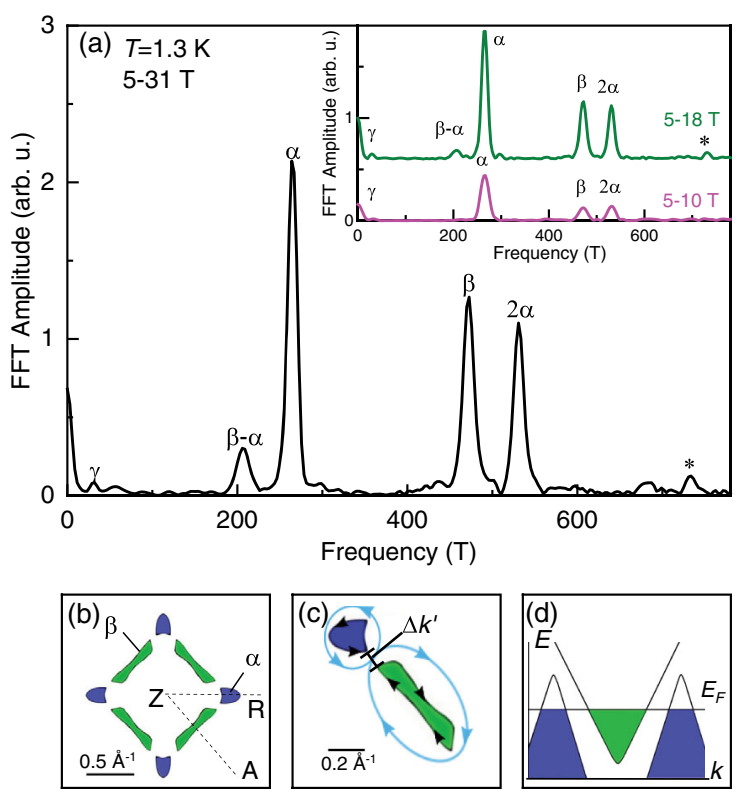

FIG. 2. Fast Fourier transform analysis of the data from 5 to $31 \mathrm{~T}$ at $T=1.3 \mathrm{~K}$. (a) Low-frequency FFT spectrum for different field ranges (inset). The FFT peaks with the individual extremal orbits are labeled as $\alpha, \beta$, and $\gamma$. The "figure of eight" orbit with the frequency $\beta-\alpha$ is also indicated. (b) Sketch of the projection of the diamond-shaped Fermi surface in the $Z-R-A$ plane in the first Brillouin zone for $\mathbf{B} \| c\left(\theta=0^{\circ}\right)$. The gap $\Delta k^{\prime}$ between the orbits is exaggerated here for illustration. The electron pockets, $\beta$, are indicated in green; the hole pockets, $\alpha$, in blue. (c) Expanded view that highlights the "figure of eight" orbit in momentum space created by an individual electron and hole orbit. The arrows indicate the direction in which charge carriers can move around the pockets, and $\Delta k^{\prime}$ represents the separation in $k$ space between the adjacent pockets. (d) Schematic of the dispersion relation $E(k)$. The horizontal line indicates $E_{F}$.

experimental variation of the frequencies with magnetic field angle $(\theta)$, as $B$ is rotated from $c$ towards $a$, to the DFT calculations (see Supplemental Material Fig. S3 [23]). The $\alpha$ orbit is close to the prediction for the hole pocket located at the vertex of the diamond-shaped Fermi surface in the $Z-R-A$ plane and is highlighted in blue in Fig. 2(b). The $\beta$ orbit has a very similar $\theta$ dependence (including the splitting for $\theta>20^{\circ}$ ) to that predicted for the electron pocket running parallel to the top rung of the nodal loop and indicated in green in Fig. 2(b). The absolute frequency $f_{\beta}$ is experimentally $\sim 25 \%$ lower than predicted.

Additionally, we observe a low-frequency orbit at $32 \mathrm{~T}$ labeled $\gamma$ (also observed in Ref. [22]), which we attribute to a closed orbit in a plane between the $Z-R-A$ and the $\Gamma-M-X$ planes. The peak at $734 \mathrm{~T}$, marked with an asterisk in Fig. 2(a), matches the sum of $\beta+\alpha$, which semiclassically should be "forbidden" because it requires the sudden reversal of the electron momentum [30].

The main finding of this work is the observation of a peak in the FFT spectrum at a frequency of $207 \mathrm{~T}$, which is consistent with the assignment $\beta-\alpha$. An orbit that includes 
these two pockets is semiclassically allowed and leads to quantum oscillations with a frequency set by the difference between the two contributing pockets [7,36-38]. In principle, such an orbit could be observed in any semimetal with adjacent electron and hole pockets due to quasiparticle tunneling [36]. However, following the theory of magnetic breakdown, such an orbit is reduced in amplitude by a factor $R_{\mathrm{MB}}=\exp \left(-B / B_{c}\right)$, where the breakdown field $B_{c} \propto \Delta k^{2}$, with $\Delta k$ being the gap between adjacent pockets of the Fermi surface $[29,36]$, that is in general too large for this peculiar effect to be observed at experimentally available magnetic fields. The phenomenon of magnetic breakdown goes back to early experiments on metals such as $\mathrm{Mg}$ and $\mathrm{Be}$ originating from electron-electron tunneling $[39,40]$.

In the context of TSMs, such an orbit has been predicted in type-II WSMs and has been described as a momentumspace counterpart of Klein tunneling [7] at a $p-n$ junction [41]. In HfSiS, the specific shape of the Fermi surface provides a similar condition for magnetic breakdown. Specifically, in the $Z-R-A$ plane, electron and hole pockets are sufficiently close for quasiparticle tunneling to occur, albeit with a finite gap between them as opposed to zero gap in the case of the type-II WSM. Therefore, a "figure of eight" orbit can be realized as sketched in Fig. 2(c). The corresponding DFT calculation in this plane and the breakdown gap are illustrated in Fig. S4 in the Supplemental Material [23]. As shown in the inset of Fig. 2(a), where we present FFT spectra calculated for different magnetic field ranges, the $\beta-\alpha$ orbit is only present once the magnetic field is larger than a critical field consistent with the picture of magnetic breakdown [36,42].

We focus now on Fig. 3, which illustrates the angle and temperature dependence of the individual $\alpha$ and $\beta$ orbits, as well as the $\beta-\alpha$ and $\beta+\alpha(*)$ orbits. Figure 3(a) shows the angle dependence at $1.3 \mathrm{~K}$ for small angles close to $\mathbf{B} \| c$. Interestingly, the $\beta-\alpha$ orbit vanishes within two degrees of misalignment between the magnetic field and the $c$ axis. This results from the rapid growth of the breakdown gap $k_{g}$ with a misalignment angle similar to the case of the type-II WSM [7] (see calculations in the Supplemental Material [23]). In Be, where electron-electron tunneling is the origin for magnetic breakdown, the oscillations are also considerably damped for small angles; however, their amplitude is found to increase again at higher angles [40].

The $\alpha$ and $\beta$ frequencies disperse in different ways as the orientation of $B$ is changed with respect to the $c$ axis. $f_{\alpha}$ is almost constant in this range of angles, whereas $f_{\beta}$ changes slightly with tilt angle and disappears between $4^{\circ}$ and $8^{\circ}$ corresponding to a so-called spin zero, where different spin split Fermi surfaces interfere. As illustrated in Fig. S3, where the evolution of the main orbits is shown together with expectations from the DFT calculations, the amplitude of the $\beta$ frequency recovers and survives over the entire angular range studied. The orbit with the "forbidden" frequency $\alpha+\beta$ disperses strongly with angle and maintains
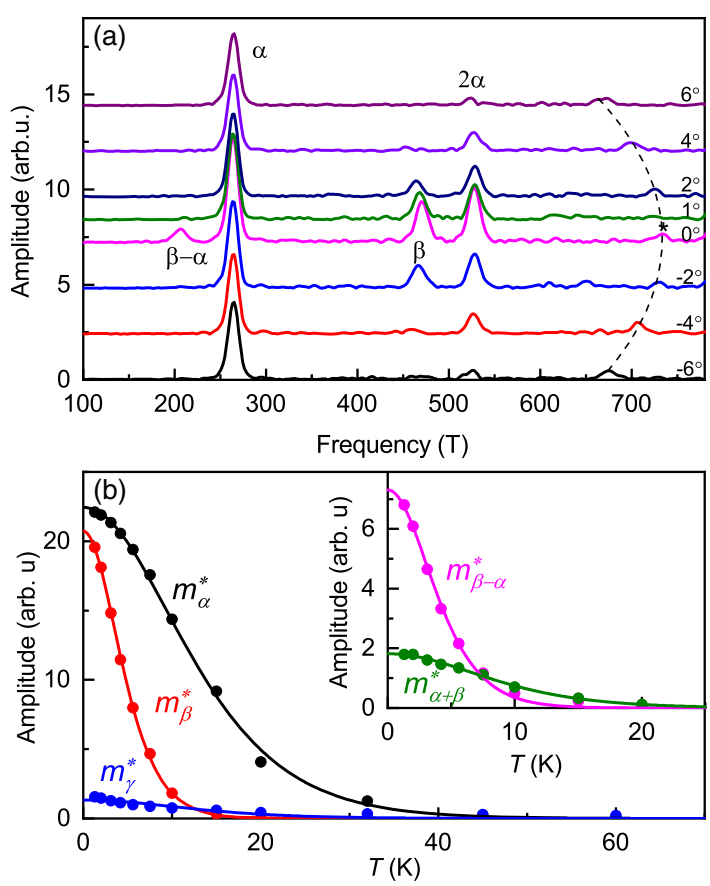

FIG. 3. (a) FFT spectra at $\mathbf{B} \| c\left(\theta=0^{\circ}\right)$ and at small tilt angles for $T=1.3 \mathrm{~K}$. While the individual orbits disperse differently with increasing tilt angle, the $\beta-\alpha$ orbit can only be observed near $\theta=0^{\circ}$. The dashed line is a guide to the eye, following the $\alpha+\beta$ (*) frequency. (b) Mass plot for the individual orbits $\alpha$ and $\beta$, and the $\beta-\alpha$ and $\alpha+\beta$ orbits (inset). The resulting cyclotron masses are specified in Table I.

a relatively high intensity, suggesting a different origin from the $\beta-\alpha$ orbit. It is possible that this orbit results from either a breakdown orbit [31,32] or magnetic interaction effects [23,29].

Figure 3(b) shows the evolution of the FFT amplitude for the individual orbits $\alpha, \beta$, and $\gamma$ with temperature. Using the standard Lifshitz-Kosevich formula for the thermal damping of the oscillations $[29,43]$, we extract the cyclotron masses $m^{*}=\left(\hbar^{2} / 2 \pi\right)\left(d A_{F} / d E\right)$, with $A$ being the crosssectional area for each of the observed orbits. The results are shown in Table I [44]. The error margins in these results

TABLE I. Observed frequencies and extracted cyclotron masses in the field range from 10 to $31 \mathrm{~T}$ from the FFT analysis [44] in comparison to DFT calculations (labeled as "Th."). $m_{e}$ is the free electron mass.

\begin{tabular}{lccccc}
\hline \hline Orbit & FFT & DFT & Plane & $\begin{array}{c}m_{c} / m_{e} \\
(\text { Exp. })\end{array}$ & $\begin{array}{c}m_{c} / m_{e} \\
(\mathrm{Th} .)\end{array}$ \\
\hline$\alpha$ & $264 \mathrm{~T}$ & $294 \mathrm{~T}$ & $Z-R-A$ & $(0.177 \pm 0.003)$ & 0.17 \\
$\beta$ & $473 \mathrm{~T}$ & $631 \mathrm{~T}$ & $Z-R-A$ & $(0.48 \pm 0.02)$ & 0.63 \\
$\gamma$ & $32 \mathrm{~T}$ & $38 \mathrm{~T}$ & in between & $(0.17 \pm 0.06)$ & $\ldots$ \\
$2 \alpha$ & $528 \mathrm{~T}$ & $\ldots$ & $Z-R-A$ & $(0.32 \pm 0.02)$ & $\ldots$ \\
$\beta-\alpha$ & $207 \mathrm{~T}$ & $\ldots$ & $Z-R-A$ & $(0.59 \pm 0.06)$ & $\ldots$ \\
$\beta+\alpha$ & $734 \mathrm{~T}$ & $\ldots$ & $\cdots$ & $(0.22 \pm 0.08)$ & $\ldots$ \\
\hline \hline
\end{tabular}



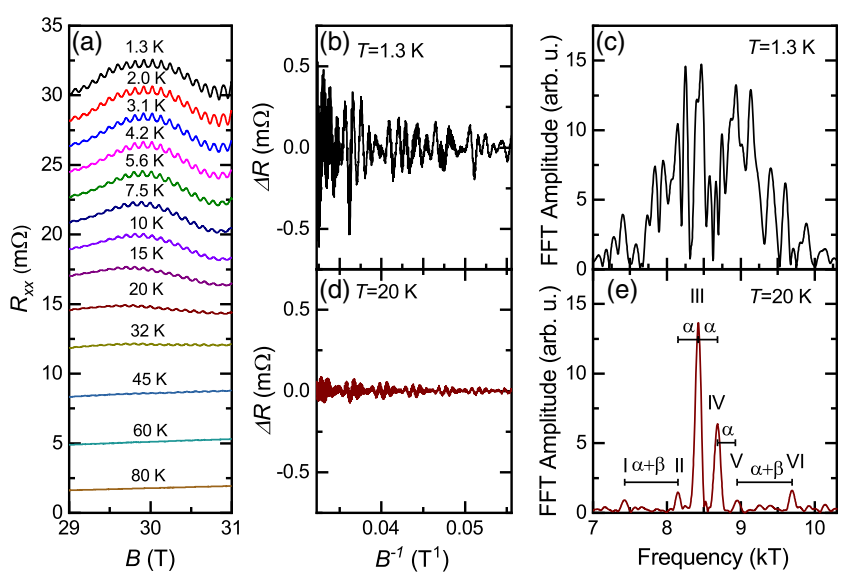

FIG. 4. High-frequency quantum oscillations. (a) $R_{x x}$ as a function of $B$ between 29 and $31 \mathrm{~T}$ for fixed temperatures ranging from 1.3 to $80 \mathrm{~K}(\mathbf{B} \| c)$. The curves are offset for clarity by $2 \mathrm{~m} \Omega$. (b), (c) and (d),(e) Oscillatory resistance $\Delta R$ versus $1 / B$ and FFT analysis for 1.3 and $20 \mathrm{~K}$, respectively. The spacer markers for the $20 \mathrm{~K}$ FFT indicate that the frequencies are separated by different combinations of the $\alpha$ and $\beta$ frequencies.

are estimated from the noise level and interference from nearby peaks or side lobes of the FFT window function, while the error from the fit function is an order of magnitude smaller. Although the cyclotron mass for the $\alpha$ pocket is in agreement with the DFT prediction, the cyclotron mass of the $\beta$ pocket is found to be smaller than predicted. The discrepancy, however, is almost perfectly proportional to the DFT overestimate of the frequency.

For the "figure of eight" orbit, one expects $\left|m_{\beta-\alpha}^{*}\right|=$ $\left|m_{\beta}^{*}\right|+\left|m_{\alpha}^{*}\right|$ [23]. Within our experimental uncertainty, we find that $m_{\beta-\alpha}^{*}$ indeed agrees with the sum of the cyclotron masses from the individual pockets involved in this breakdown orbit. These findings, the frequency $\beta-\alpha$ in the FFT, and the extracted mass $m_{\beta-\alpha}^{*}$ are all consistent with theoretical predictions for Klein tunneling $[7,36]$. Assuming that the classical forbidden orbit $\beta+\alpha$ is indeed formed by two pockets with electron- and hole-like character, $m_{\beta+\alpha}^{*}=\left|m_{\beta}^{*}\right|-\left|m_{\alpha}^{*}\right|$ again, in good agreement with our observation. However, this would require a mechanism reversing the direction of circulation of carriers around a closed orbit while preserving the sign of $d A / d E$. Despite the agreement concerning $m_{\beta+\alpha}^{*}$, we emphasize that the dispersion of $\beta+\alpha(*)$ shown in Fig. 3(a) is different from the angle dependence of the individual $\alpha$ and $\beta$ pockets. Thus, the origin of the $\beta+\alpha$ orbit demands further investigation [45].

Finally, we turn to the high-frequency oscillations that appear above $15 \mathrm{~T}$ in Fig. 1. Figure 4(a) shows an enlarged view of these oscillations between 29 and $31 \mathrm{~T}$ for different temperatures. By removing the background that originates from the MR as well as from the low-frequency oscillations discussed so far, we find a complex oscillatory pattern in $\Delta R$ as a function of the inverse magnetic field, $B^{-1}$, shown for 1.3 and $20 \mathrm{~K}$ in Figs. 4(b) and 4(d). The corresponding spectra of the FFT then comprise a series of peaks ranging from 7 to around $10 \mathrm{kT}$, with the most pronounced ones around 8 and $9 \mathrm{kT}$. There are no individual orbits in the DFT-derived Fermi surface with areas that match such high frequencies. Thus, we attribute these peaks in the FFT spectrum to magnetic breakdown orbits that encircle the entire "diamond" in the Z-R-A-plane; see Fig. 2(b). These orbits are also strongly suppressed for small-tilt angles away from the $c$ axis, as found in $\mathrm{ZrSiS}$ [8].

Unfortunately, the resolution of the low-temperature FFT spectra of this high-frequency part of our data does not allow us to investigate individual peaks in detail, as their close spacing leads to a considerable overlap of adjacent peaks. At $20 \mathrm{~K}$, however, most of the frequencies have vanished, and only six peaks remain in the spectrum; see Fig. 4(e). Two of these peaks (III and IV) have an amplitude that is almost as large as at $1.3 \mathrm{~K}$, while the other four peaks are significantly damped. This also precludes our ability to investigate the $T$ dependence of the quantum oscillation amplitude that in $\mathrm{ZrSiS}$ showed anomalous behavior [8] and requires further detailed investigation. The peaks II-V are all spaced in frequency by $264 \mathrm{~T}$ (i.e., the frequency of the orbit around the $\alpha$ pocket), whereas the peaks I and VI are a distance of approximately $734 \mathrm{~T}(\alpha+\beta)$ away. This suggests that these peaks correspond to orbits around the Fermi surface that incorporate different multiples of the $\alpha$ and $\beta$ pockets.

In conclusion, we have demonstrated a "figure of eight" orbit in the gapped NLSM HfSiS, realizing an analogue of the momentum-space Klein tunneling proposed for gapless type-II WSMs. This special type of magnetic breakdown is only present if the magnetic field is oriented almost exactly parallel to the $c$ axis and its cyclotron mass is found to be the sum of the masses of the individual electron and hole pockets. Although breakdown has been extensively studied in simple elements, and in organic metals, this special type of magnetic breakdown between electron and hole pockets has not been observed previously. The reason is that in compensated organic metals, the Fermi surface is always strongly two dimensional, so that the areas of the electron and hole pockets are exactly equal at all $k_{z}$; hence the difference frequency is always zero and unobservable. The observation in the NLSM HfSiS implies that the phenomenon of momentum-space Klein tunneling is not confined to type-II WSMs. Rather, our results suggest that this is a generic property of semimetals with adjacent electron and hole pockets, provided that the applied magnetic field is strong enough to overcome their $k$-space separation.

This work was supported by the High Field Magnet Laboratory at Radboud University/Foundation for Fundamental Research on Matter (HMFL-RU/FOM), a member of the European Magnetic Field Laboratory, and 
by the United Kingdom Engineering and Physical Sciences Research Council (Grant No. EP/R011141/1). We thank Tom O'Brien, Stephen Hayden, and Mark Kartsovnik for helpful discussions.

*Present address: Center for Nanotechnology Innovation@ NEST, Istituto Italiano di Tecnologia, P.za S. Silvestro 12, 56127 Pisa, Italy.

†steffen.wiedmann@ru.nl

[1] A. A. Burkov, Nat. Mater. 15, 1145 (2016).

[2] N. P. Armitage, E. J. Mele, and A. Vishwanath, Rev. Mod. Phys. 90, 015001 (2018).

[3] X. Wan, A. M. Turner, A. Vishwanath, and S. Y. Savrasov, Phys. Rev. B 83, 205101 (2011).

[4] A. C. Potter, I. Kimchi, and A. Vishwanath, Nat. Commun. 5, 5161 (2014).

[5] H. B. Nielsen and M. Ninomiya, Phys. Lett. B 130, 389 (1983).

[6] P. J. W. Moll, N. L. Nair, T. Helm, A. C. Potter, I. Kimchi, A. Vishwanath, and J. G. Analytis, Nature (London) 535, 266 (2016).

[7] T. E. O'Brien, M. Diez, and C. W. J. Beenakker, Phys. Rev. Lett. 116, 236401 (2016).

[8] S. Pezzini, M. R. van Delft, L. M. Schoop, B. V. Lotsch, A. Carrington, M. I. Katsnelson, N. E. Hussey, and S. Wiedmann, Nat. Phys. 14, 178 (2018).

[9] C. Fang, H. Weng, Xi Dai, and Z. Fang, Chin. Phys. B 25, 117106 (2016).

[10] G. Bian et al., Nat. Commun. 7, 10556 (2016).

[11] Y. Wu, L.-L. Wang, E. Mun, D. D. Johnson, D. Mou, L. Huang, Y. Lee, S. L. Budko, P. C. Caneld, and A. Kaminski, Nat. Phys. 12, 667 (2016).

[12] M. Neupane, I. Belopolski, M. M. Hosen, D. S. Sanchez, R. Sankar, M. Szlawska, S.-Y. Xu, K. Dimitri, N. Dhakal, P. Maldonado, P. M. Oppeneer, D. Kaczorowski, F. Chou, M. Z. Hasan, and T. Durakiewicz, Phys. Rev. B 93, 201104(R) (2016).

[13] L. M. Schoop, M. N. Ali, C. Straßer, A. Topp, A. Varykhalov, D. Marchenko, V. Duppel, S. S. P. Parkin, B. V. Lotsch, and C. R. Ast, Nat. Commun. 7, 11696 (2016).

[14] X. Wang, X. Pan, M. Gao, J. Yu, J. Jiang, J. Zhang, H. Zuo, M. Zhang, Z. Wei, W. Niu, Z. Xia, X. Wan, Y. Chen, F. Song, Y. Xu, B. Wang, G. Wang, and R. Zhang, Adv. Electron. Mater. 2, 1600228 (2016).

[15] M. N. Ali, L. Schoop, C. Garg, J. M. Lippmann, E. Lara, B. Lotsch, and S. Parkin, Sci. Adv. 2, e1601742 (2016).

[16] J. Hu, Z. Tang, J. Liu, Y. Zhu, J. Wei, and Z. Mao, Phys. Rev. B 96, 045127 (2017).

[17] R. Singha, A. Pariari, B. Satpati, and P. Mandal, Proc. Natl. Acad. Sci. U.S.A. 114, 2468 (2017).

[18] J. Hu, Z. Tang, J. Liu, X. Liu, Y. Zhu, D. Graf, K. Myhro, S. Tran, C.-N. Lau, J. Wei, and Z. Mao, Phys. Rev. Lett. 117, 016602 (2016).

[19] A. Topp, J. M. Lippmann, A. Varykhalov, V. Duppel, B. V. Lotsch, C. R. Ast, and L. M. Schoop, New J. Phys. 18, 125014 (2016).
[20] D. Takane, Z. Wang, S. Souma, K. Nakayama, C. X. Trang, T. Sato, T. Takahashi, and Y. Ando, Phys. Rev. B 94, 121108 (2016).

[21] C. Chen et al., Phys. Rev. B 95, 125126 (2017).

[22] N. Kumar, K. Manna, Y. Qi, S.-C. Wu, L. Wang, B. Yan, C. Felser, and C. Shekhar, Phys. Rev. B 95, 121109(R) (2017).

[23] See Supplemental Material at http://link.aps.org/ supplemental/10.1103/PhysRevLett.121.256602 for electronic structure calculations, additional data and analysis on the angle dependence, technical details of the sample synthesis, preparation and characterization, and a discussion about magnetic interaction, which includes Refs. [24-27].

[24] H. Onken, Naturwissenschaften 51, 334 (1964).

[25] P. Blaha, K. Schwarz, G. K. H. Madsen, D. Kvasnicka, and J. Luitz, WIEN2k (Karlheinz Schwarz, Techn. Universität Wien, Austria, 2001).

[26] J. P. Perdew, K. Burke, and M. Ernzerhof, Phys. Rev. Lett. 77, 3865 (1996).

[27] J.-M. Carter, D. Podolsky, and H.-Y. Kee, Phys. Rev. B 81, 064519 (2010).

[28] M. B. Schilling, L. M. Schoop, B. V. Lotsch, M. Dressel, and A. V. Pronin, Phys. Rev. Lett. 119, 187401 (2017).

[29] D. Shoenberg, Magnetic Oscillations in Metals (Cambridge University Press, Cambridge, England, 1984).

[30] "Forbidden" frequencies have been observed in the context of magnetic breakdown in magnesium [31] and organic conductors [32] and might be caused, e.g., by oscillations in the chemical potential $[33,34]$ or $B$-dependent oscillation damping [35].

[31] J. W. Eddy and R. W. Stark, Phys. Rev. Lett. 48, 275 (1982).

[32] F. A. Meyer, E. Steep, W. Biberacher, P. Christ, A. Lerf, A. G. M. Jansen, W. Joss, P. Wyder, and K. Andres, Europhys. Lett. 32, 681 (1995).

[33] N. Harrison, J. Caulfield, J. Singleton, P. H. P. Reinders, F. Herlach, W. Hayes, M. Kurmoo, and P. Day, J. Phys. Condens. Matter 8, 5415 (1996).

[34] J. Y. Fortin and T. Ziman, Phys. Rev. Lett. 80, 3117 (1998).

[35] V. M. Gvozdikov, Y. V. Pershin, E. Steep, A. G. M. Jansen, and P. Wyder, Phys. Rev. B 65, 165102 (2002).

[36] M. I. Kaganov and A. A. Slutskin, Phys. Rep. 98, 189 (1983).

[37] A. Alexandradinata and L. Glazman, Phys. Rev. Lett. 119, 256601 (2017).

[38] A. Alexandradinata and L. Glazman, Phys. Rev. B 97, 144422 (2018).

[39] M. G. Priestley, L. M. Falicov, and G. Weisz, Phys. Rev. 131, 617 (1963).

[40] N. E. Alekseevskii, A. A. Slutskin, and V. S. Egorov, J. Low Temp. Phys. 5, 377 (1971).

[41] M. I. Katsnelson, K. S. Novoselov, and A. K. Geim, Nat. Phys. 2, 620 (2006).

[42] M. H. Cohen and L. M. Falicov, Phys. Rev. Lett. 7, 231 (1961).

[43] I. M. Lifshitz and A. M. Kosevich, Sov. Phys. J. Exp. Theor. Phys. 2, 639 (1953).

[44] The experimental masses were determined by fitting the amplitude of the FFT peak versus temperature assuming an effective average field equal to the average inverse field in the window. A more refined procedure, taking into account 
the finite Dingle term, would increase the $\alpha$ and $\beta$ masses by $8 \%$ and $15 \%$, respectively.

[45] The masses of forbidden frequencies tend to be far off from the theoretically expected values in organic conductors $[46,47]$. It is also interesting to note that the masses associated with $\beta-\alpha$ and $2 \alpha$ are by a factor of 0.9 smaller than predicted by the Lifshitz-Kosevich theory. This might be related to the $2 \mathrm{D}$ character of the $\alpha$ pocket, causing enhanced oscillations in the chemical potential neglected in the Lifshitz-Kosevich theory $[48,49]$, an effect that has been discussed as a possible origin of mass deviations in quasi2D organic conductors $[32,47]$.

[46] M. V. Kartsovnik, G. Yu. Logvenov, T. Ishiguro, W. Biberacher, H. Anzai, and N. D. Kushch, Phys. Rev. Lett. 77, 2530 (1996).

[47] S. Uji, M. Chaparala, S. Hill, P. S. Sandhu, J. Quails, L. Seger, and J. S. Brooks, Synth. Met. 85, 1573 (1997).

[48] K. Kishigi and Y. Hasegawa, Phys. Rev. B 72, 045410 (2005).

[49] M. A. Itskovsky, Phys. Rev. B 68, 054423 (2003). 